\title{
Modelling of a Filter using EMI/EMC Considerations
}

\author{
I. Vinaychowdary \\ Department of ECE, \\ Gitam University
}

\author{
Ch R Phani Kumar \\ Department of ECE, \\ Gitam University
}

\begin{abstract}
There are so many Filters are available in the nature of communication. The modern communications are purely depends on the diversity of the signals. The signaling systems are fairy major problem with the noise that occurs internally or externally. The standard measuring of electromagnetic pulse problems can reduce the problems of noise in the modern digital systems. The present work is to overcome the problems of noise that occurs internally or externally and malfunctions of the circuit with the measurement of EMI/EMC considerations. In Digital Signal Processing system consists of devices such as Phase Shifters and Power Dividers and Filters as well as Multiplexers which are used to transmit and adjust the amplitude and phase of the electrical signals in such a way is to form desire signal. After forming the desire beams of a system and then malfunctions of the system with the measurement of EMI/EMC consideration and observes the beams without any noise in the Digital signal processing system and compared both performances. By using the stimulation and synthesis can be carried out to Digital Signal processing System. The different standard methods to reduce insertion loss are proposed to measure the noise source impedance to particular systems. The information obtained through the proposed method enables the prediction of EMI filter performance and the design of a suitable filter for a switch mode power supply.
\end{abstract}

\section{Keywords}

Insertion Loss, EMI Filters, EMI/EMC Standards.

\section{INTRODUCTION}

The Electromagnetic environment is an integral part of the world in which we live. The electromagnetic environment created by these intentional and unintentional sources, when sufficiently strong, interference with the operation of many electrical and electronics equiptment and systems. Noise is any electrical signal present in a circuit other than the desired signal. An electromagnetic disturbance is any electromagnetic phenomenon which may degrade the performance of a device, or an equipment, or a system [1]. The electromagnetic disturbance can be in the nature of an electromagnetic noise, or an unwanted signal, or a change in the propagation medium itself. Electromagnetic interference (EMI) is the degradtion in the performance of a device, or an equipment, or a system caused by an electromagnetic disturbance [2]

Electromagnetic compatibility (EMC) is the ability of an electronic system to (1) function properly in its intended electromagnetic environment and (2) not be a source of pollution to that electromagnetic environment. The electromagnetic environment is composed of both radiated and conducted energy. EMC therefore has two aspects, emission and susceptibility. Susceptibility is the capability of a device or circuit to respond to unwanted electromagnetic energy (i.e., noise). The opposite of susceptibility is immunity. The immunity level of a circuit or device is the electromagnetic environment in which the equipment can operate satisfactorily, without degradation, and with a defined margin of safety. One difficulty in determining immunity (or susceptibility) levels is defining what constitutes performance degradation[3]. To guarantee that EMC is a consideration in the design of all electronic products, various government agencies and regulatory bodies have imposed EMC regulations that a product must meet before it can be marketed. These regulations control allowable emissions and in some cases define the degree of immunity required.

All electronic equipment produced today includes EMI filtering circuits. Likewise, all switch-mode power supplies have internal EMI filters. However, there are circumstances where the EMI filters within these electronic devices require a supplemental filter to meet more stringent electrical noise regulations or to protect the device from excessive external noise sources [4]. EMI can be in form of conducted EMI, which means the noise travels along electrical conductors, wires, printed-circuit traces, or electronic components such as transformers, inductors, capacitors, semiconductors, and resistors. Electrical noise can also be in the form of radiated EMI (RFI), noise that travels through the air or free space as magnetic fields or radio waves. RFI is usually controlled by providing metal shielding that contains the magnetic fields or radio waves within the equipment's enclosure [5]. EMI filtering circuits are employed so the end product complies with applicable EMC standards. Among the most frequently cited EMC standards are EN55022 for IT equipment, EN55011 for industrial equipment, and, in the U.S., FCC Class A for commercial or industrial equipment or FCC Class $\mathrm{B}$ for residential equipment. FCC Class B is tougher and more restrictive than Class A. For the majority of these standards, the conducted EMI frequency range is usually defined as being between $150 \mathrm{kHz}$ and $30 \mathrm{MHz}$, as measured by a spectrum analyzer. In some cases, this range begins as low as $10 \mathrm{kHz}$. By comparison, RFI is usually defined to range from $30 \mathrm{MHz}$ to $1 \mathrm{GHz}$. In either power supplies or electronic equipment, it is the function of the EMI filter to keep any internally generated noise contained within the device and to prevent any external ac line noise from entering the device [6]. EMI filters usually comprise a network of passive electronic components including capacitors and inductors that form L-C circuits. Since unwanted EMI is at much higher frequencies than normal signals, the EMI filter works by selectively blocking or shunting unwanted higher frequencies. Basically, the inductive part of the EMI filter is designed to act as a low-frequency pass device for the ac line frequencies and a high-frequency blocking device [7]. Other parts of the EMI filter use capacitors to bypass or shunt unwanted highfrequency noise away from the sensitive circuits. The net result is that the EMI filter significantly reduces or attenuates any unwanted noise signals from entering or leaving the protected electronic device [8]. 
Many specs and ratings must be considered when selecting EMI filters. These include case size, I/O connections, mounting type, safety agency approvals, operating voltage, operating current (ac or dc amps), leakage current, isolation resistance, withstand test voltages, high-voltage pulse or spike attenuation, operating temperature range, dc resistance, and insertion loss [9]. For medical applications, the installed leakage current and withstand test voltages of the final assembly are important parameters for meeting the EMC requirements relative to patient safety. Insertion loss information for an EMI filter is usually presented in the form of graphs, plots, or tables that show how well the EMI filter attenuates or suppresses the conducted differential and common-mode noise within its operating range [10]. As mentioned previously, the usual frequency range specified in most EMC standards for conducted EMI emissions is from $150 \mathrm{kHz}$ to $30 \mathrm{MHz}$. To confirm that an electronic device meets the limits of a specific standard, it must be tested with a spectrum analyzer and a line impedance stabilization network (LISN). Ac power is routed through the LISN to the device under test. The LISN standardizes the measurement impedance to $50 \Omega$ and provides an isolated RF output to a spectrum analyzer, which provides a plot of the conducted emissions coming from the device [11].

\section{PROCEDURE}

The basic circuit test analysis has been carried out by using different EMI Filters. And the performance and the simulation results have been analysed using the different EMI/EMC Standards. The analysis has been carried by using the MILSTD $220 \mathrm{~A}$ by calculating the Insertion Losses using their standards and testing procedure can be carried out Using MIL-STD 220B and MIL-STD 220C to stimulate the exact quality signal without any sort of Interference.

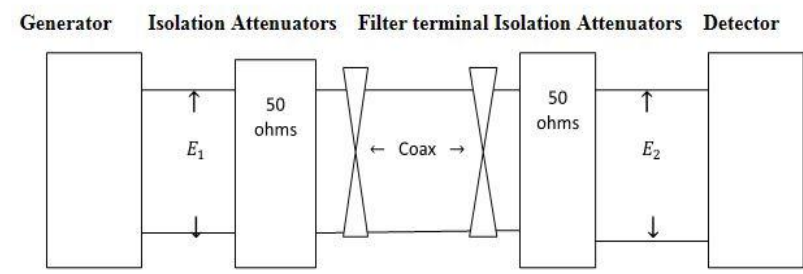

Fig.1 MIL-STD-220A filters insertion loss measurement equipment before filter insertion.

\section{ANALYSIS FILTER USING THE EMI/EMC STANDARDS}

In this section, the performances for different EMI Filters were analyzed. Section 3.1 Analysis based on Insertion Loss using MIL-STD 220A and Section 3.2 Test analysis of different components/parameters using MIL-STD 220B and MIL-STD 220C.

\subsection{Analysis based on Insertion Loss using MIL-STD 220A:}

In MIL-STD-220A, the insertion loss of a filter in a transmission system at a given frequency is defined as the ratio of voltages appearing across the line immediately beyond the point of insertion, before and after insertion. It is, however, measured as the ratio of the input voltages required to obtain a constant output voltage, prior to and after insertion of the filter between two matched isolation attenuators in a specified $50 \Omega$ transmission system.

Insertion Loss $=20 \log \left|\frac{E_{1}^{\prime}}{E_{1}}\right|$
Where $E_{1}^{\prime}$ Output voltage of the signal generator with the filter in the circuit, and $E_{1}$ Output voltage of the signal generator with the filter not in the circuit

For the filter operating between arbitrary generator and load impedances

$$
\begin{aligned}
& \text { Insertion Loss }=20 \log \left|\frac{E_{2}^{\prime}}{E_{2}}\right|= \\
& 20 \log \left|\frac{z_{O 2}}{\sqrt{Z_{O 1}\left(Z_{O 2}-Z_{S 1}\right)}}\right| \cdot\left|\frac{z_{G}+\frac{z_{G} Z_{L}}{Z_{O 2}}+Z_{s 1}+\frac{z_{L} z_{O 1}}{Z_{O 2}}}{Z_{L}+Z_{G}}\right|
\end{aligned}
$$

Where $Z_{L}$ Impedance of the load; $Z_{G}$ Impedance of the generator; $Z_{s 1}$ Input impedance at terminal 1 with terminal 2 shorted; $Z_{o 1}$ Input impedance at terminal 1 with terminal 2 open; $Z_{s 2}$ Input impedance at terminal 2 with terminal 1 shorted; and $Z_{o 2}$ Input impedance at terminal 2 with terminal 1 open.

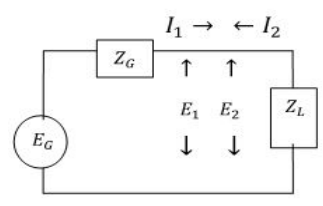

(a)

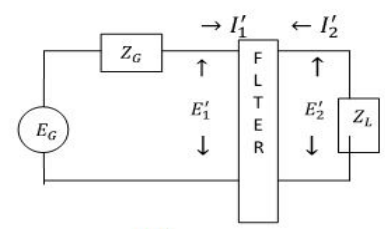

(b)
Fig: 2 Circuit used in defining the insertion voltages and currents (a) before filter insertion and (b) after filter insertion.

The final form of the MIL-STD-220A insertion-loss relation defined by (3.1) in terms of the load, and the filter short and open-circuit parameters becomes

$$
\begin{gathered}
\text { INSERTION LOSS }= \\
20 \log \left|\frac{z_{O 2}}{\sqrt{z_{O 1}\left(Z_{O 2}-Z_{S 2}\right)}}\right|\left|\left[\left(\frac{1}{z_{o 2}}+\frac{1}{z_{L}}\right) Z_{o 1}\right]\right|
\end{gathered}
$$

A comparison of (3.2) and (3.3) indicates that, except for the common

factor

$\frac{Z_{o 2}}{\sqrt{Z_{o 1}\left(Z_{o 2}-Z_{S 2}\right)}}$

there is little resemblance between the two forms of equations. In particular, it is interesting to observe that insertion loss by the classical equation is a function of the generator impedance as well as load impedance and other characteristics of the network. However, no such dependence on generator impedance exists in (3.3), making an important difference between the two insertion-loss relations. The analysis the Filter for Insertion oss measurement equivalent circuit is below.

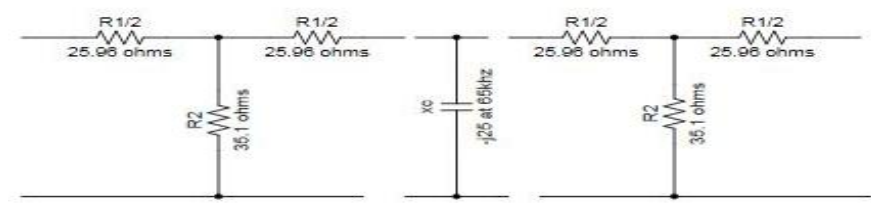

Fig: 3 MIL-STD-220A equivalent circuits for insertion loss measurement of a feedthrough capacitor. 
From Fig. 3, the open- and short-circuit impedances at $\mathrm{f}=65$ $\mathrm{kHz}$ are

$$
\begin{aligned}
& Z_{o 1}=44.96-\mathrm{J} 4.93=Z_{o 2} \\
& Z_{s 1}=45.02-\mathrm{J} 4.05=Z_{s 2}
\end{aligned}
$$

For the values of the above parameters in (3.3), the Insertion loss is

Insertion Loss $=20 \log \left|\frac{E_{2}^{\prime}}{E_{2}}\right|=23.10 \mathrm{~dB}$

From the MIL-STD-220A insertion-loss equation (3.3), the insertion loss is

$$
\text { Insertion Loss }=20 \log \left|\frac{E_{1}^{\prime}}{E_{1}}\right|=22.67 \mathrm{~dB} \text {. }
$$

The Insertion Loss for different Emi Filters has been calculated and based on this results the performance of a Filter been analyzed.

\subsection{Test Analysis of Different Components \\ Using MIL-STD 220B and MIL-STD 220C}

Filters measured by this method MIL-STD-220B are typically feed-through types, having the live conductor(s) passing through the filter providing both input and output terminals protruding from and insulated from the case which acts as the ground terminal. These filters typically contain capacitors only or capacitors and inductors, and may also contain resistors or diodes. The filters measured to this standard are normally designed for bulkhead mounting, where the input and output terminals are completely isolated from each other by the bulkhead. At a given frequency, the insertion loss of a feed through suppression capacitor or a filter connected into a given transmission system is defined as the ratio of voltages appearing across the line immediately beyond the point of insertion, before and after insertion. As measured herein, insertion loss is represented as the ratio of input voltage required to obtain constant output voltage, with and without the component, in the specified $50 \mathrm{ohm}$ system.

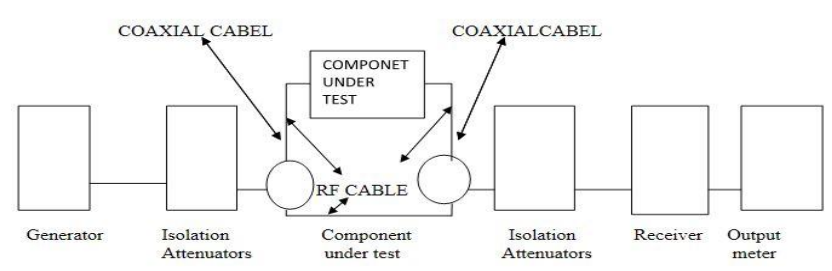

Fig: 4 Test circuit for Rapid Measurement in MIL-STD 220B.

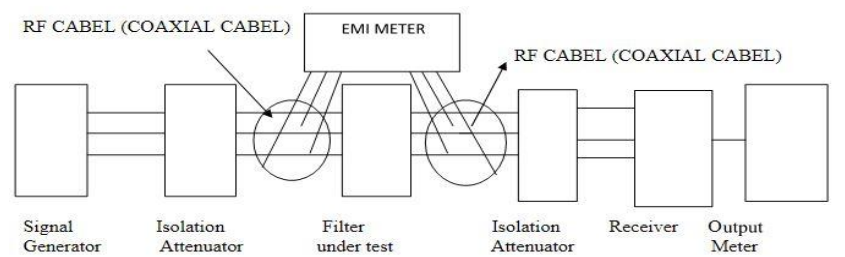

Fig: 5 Test circuit for Rapid Measurement with full Load using MIL-STD 220B.

\subsection{Analysis Using MIL-STD 220C}

Complete characterization of filters is typically achieved with sweep- frequency measurements. The most commonly measured filter characteristics are insertion loss and bandwidth. Another common measured parameter is out-ofband rejection. This is a measure of how well a filter passes signals within its bandwidth while simultaneously rejecting signals well outside that same bandwidth. The errors can be rectified using this MIL-STD 220C.

This standard is intended to provide methods for insertion loss measurements of EMI Filters and single and multiple-circuit, radio frequency power line filters. These insertion loss measurements are conducted in a $50 \mathrm{ohm}$ system for the purpose of quality control during quantity production.

\section{RESULTS}

The basic circuit analysis has been carried out using the different EMI Filters and the simulation results can be analyzed.

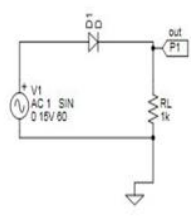

(A) Basic circuit

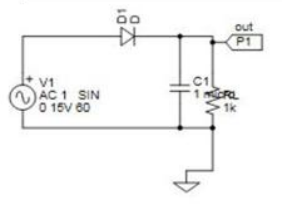

(B) Capacitive Filter circuit

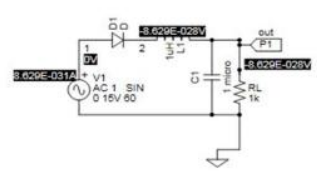

(C) LC Filter circuit

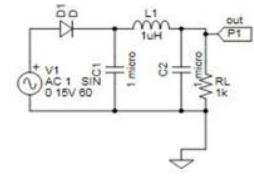

(D) $\pi$-Filter circuit

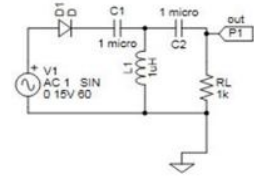

(E) T-Section Filter circuit
Fig: 6 Basic circuit analyses using different EMI Filters using Spice simulation.

The simulation results for the analysis using spice
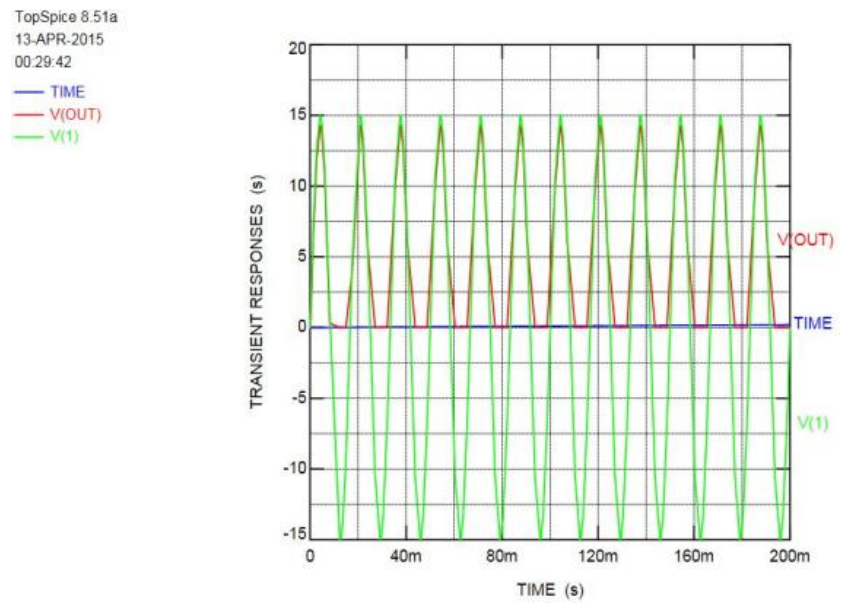

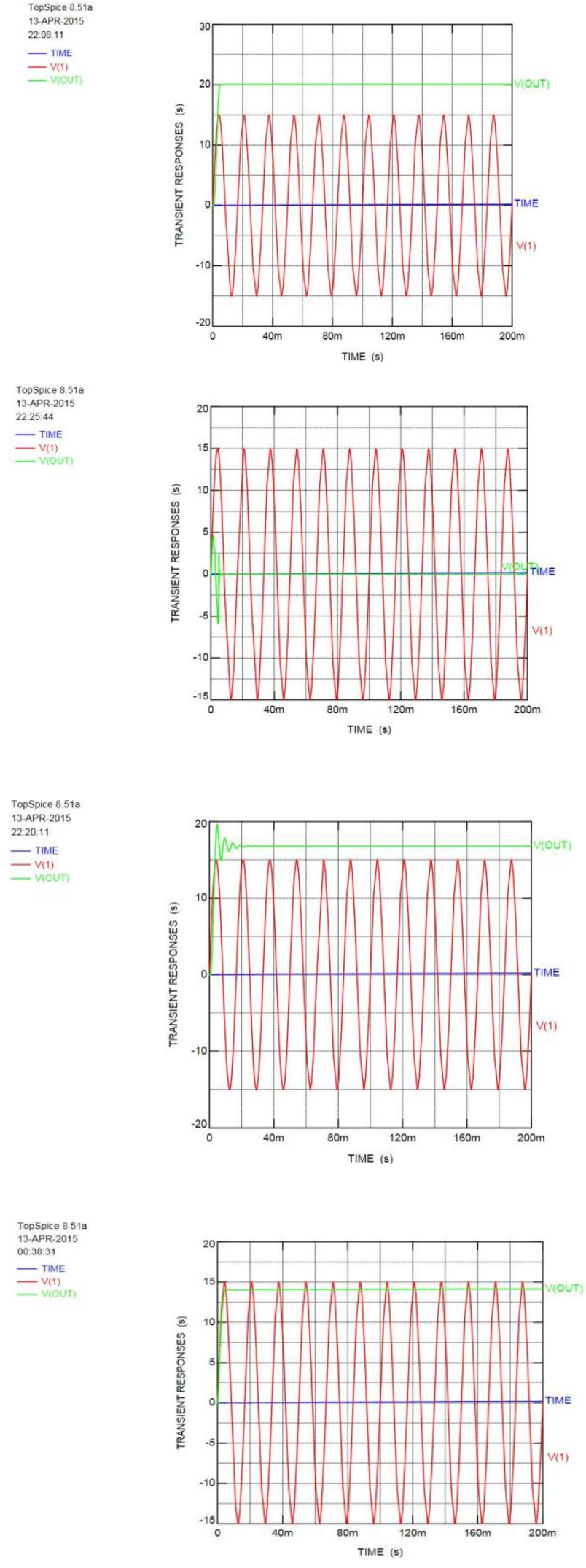

Fig: 7 EMI Filters using Spice simulation.

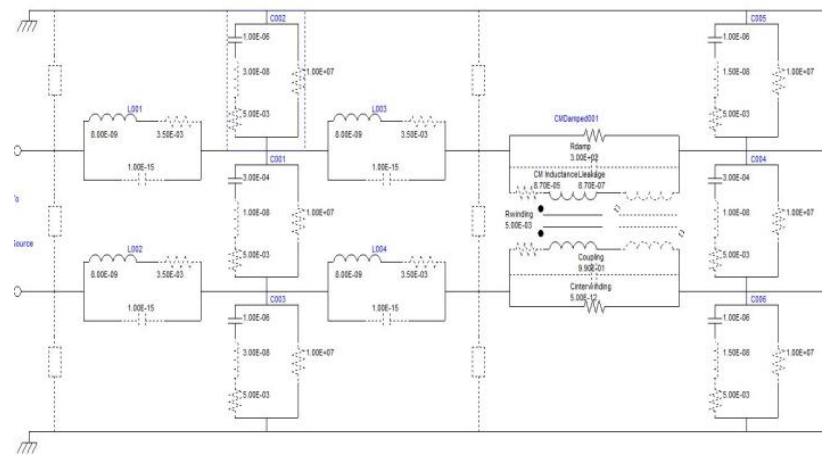

Figure: 8 Design Filter circuit

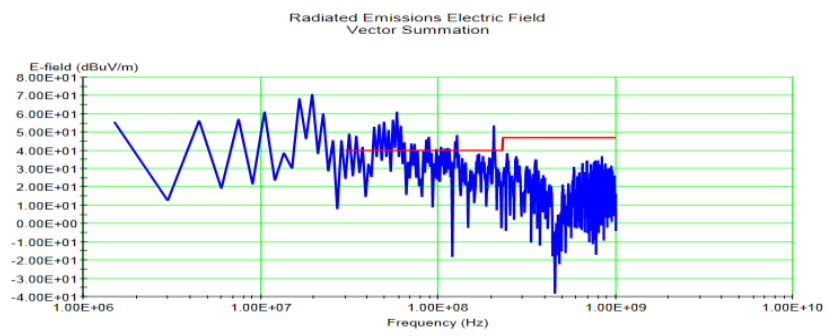

Figure: 9 The output of a Filter using the EMI Analyser.

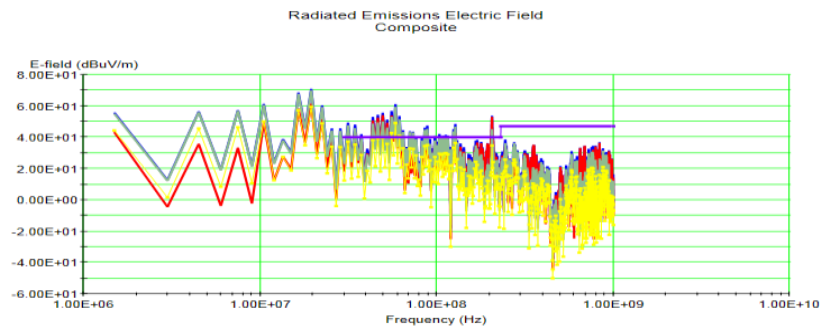

Figure: 10 The output of Composite simulation of a Filter using EMI Analyzer.

\section{CONCLUSION}

A In this paper, the signals can be analyzed by using different techniques and getting exact output but, still there are some sorts of interferences generated while transferring the signal to the desire point. These interferences are due to some parasitic elements have great effect on the high frequency characteristic although its value is very small, while others have little effect on it. And to overcome this interferences occur due to insertion loss are reduced by applying EMI/EMC standards and techniques. In MIT-STD the testing of filter can be carried out and measurements can be taken using EMI Meter and power dissipations can be explained. By using MIT-STD the errors in filters can be rectified and provide a quality signal without any interference. This thesis work is concentrated only on analysis of different Filters and calculations been done for verification and simulation results been taken into consideration by using the EMC standard and mainly focus on amount of reduction of EMI occur from different SMPS. Further it can also been developed a hardware and test been processes using Spectrum Analyzers to observe the performance of different systems or SMPS. 


\section{REFERENCES}

[1] Bishnoi, H.; Baisden, A.C.; Mattavelli, P.; Boroyevich, D. "Analysis of EMI Terminal Modeling of Switched Power Converters", Power Electronics, IEEE Transactions on, On page(s): 3924 - 3933 Volume: 27, Issue: 9, Sept. 2012.

[2] Shuo Wang; Lee, F.C.; Odendaal, W.G. "Using scattering parameters to characterize EMI filters", Power Electronics Specialists Conference, 2004. PESC 04 2004 IEEE 35th Annual, On page(s): 297 - 303 Vol.1 Volume: 1, 20-25 June 2004.

[3] Meng Jin; Ma Weiming "A new technique for modeling and analysis of mixed-mode conducted EMI noise", Power Electronics Specialists Conference, 2004. PESC 04. 2004 IEEE 35th Annual, On page(s): 1034 - 1040 Vol.2 Volume: 2, 20-25 June 2004.

[4] Meng Jin; Ma Weiming; Zhang Lei "Determination of noise source and impedance for conducted EMI prediction of power converters by lumped circuit models", Power Electronics Specialists Conference, 2004. PESC 04. 2004 IEEE 35th Annual, On page(s): 3028 - 3033 Vol.4 Volume: 4, 2004.

[5] Liu, Q.; Shen, W.; Wang, F.; Boroyevich, D.; Stefanovic, V.; Arpilliere, M. "Experimental evaluation of IGBTs for characterizing and modeling conducted EMI emission in PWM inverters", Power Electronics Specialist Conference, 2003. PESC '03. 2003 IEEE 34th Annual, On page(s): 1951 - 1956 vol.4 Volume: 4, 15-19 June 2003.
[6] Baisden, A.C.; Boroyevich, D.; van Wyk, J.D. "High Frequency Modeling of a Converter with an RF-EMI Filter", Industry Applications Conference, 2006. 41st IAS Annual Meeting. Conference Record of the 2006 IEEE, On page(s): 2290 - 2295 Volume: 5, 8-12 Oct. 2006.

[7] Sanchez, A.-M.; Regue, J.-R.; Ribo, M.; Perez, A.; Rodriguez-Cepeda, P.; Pajares, F.-J. "Automated PowerLine Filter Design Under High $50-\mathrm{Hz}$ Current Load Conditions", Electromagnetic Compatibility, IEEE Transactions on, On page(s): 717 - 724 Volume: 55, Issue: 4, Aug. 2013.

[8] Hong Li; Zhong Li; Bo Zhang; Tang, W.K.S.; Wolfgang Halang "Suppressing electromagnetic interference in direct current converters", Circuits and Systems Magazine, IEEE, On page(s): 10 - 28 Volume: 9, Issue: 4, Fourth Quarter 2009.

[9] Crosato, M.O.; Hofsajer, I.W. "Minimising conducted common mode EMI by charge balancing in a nonisolated DC-DC converter", Power Electronics Specialists Conference, 2004. PESC 04. 2004 IEEE 35th Annual, On page(s): 3146 - 3151 Vol.4 Volume: 4, 2004.

[10] Neugebauer, T.C.; Perreault, D.J. "Parasitic capacitance cancellation in filter inductors", Power Electronics Specialists Conference, 2004. PESC 04. 2004 IEEE 35th Annual, On page(s): 3102 - 3107 Vol.4 Volume: 4, 2004

[11] Shoyama, M.; Li, G.; Ninomiya, T. "Balanced switching converter to reduce common-mode conducted noise", Industrial Electronics, IEEE Transactions on, On page(s): 1095 - 1099 Volume: 50, Issue: 6, Dec. 2003. 